\title{
The Activity of Human Mirror Neurons during Observation and Time Perception
}

DOI: $10.17691 / \mathrm{stm} 2019.11 .1 .08$

Received October 25, 2018

Yu.V. Bushov, DSc, Professor, Head of the Department of Human and Animal Physiology

M.V. Svetlik, PhD, Associate Professor, Department of Medical and Biological Cybernetics ${ }^{2}$ Associate Professor, Department of Human and Animal Physiology ${ }^{1}$;

E.A. Esipenko, PhD, Associate Professor, Department of Genetic and Clinical Psychology

S.I. Kartashov, Research Engineer ${ }^{3}$; Engineer ${ }^{4}$;

V.A. Orlov, Research Engineer;

V.L. Ushakov, PhD, Associate Professor, Leading Researcher ${ }^{3}$; Senior Researcher ${ }^{4}$

${ }^{1}$ Tomsk State University, 36 Lenin Avenue, Tomsk, 634050, Russia;

${ }^{2}$ Siberian State Medical University, 2 Moscovsky Trakt, Tomsk, 634050, Russia;

${ }^{3}$ National Research Center "Kurchatov Institute", 1 Akademika Kurchatova Square, Moscow, 123182, Russia;

${ }^{4}$ National Research Nuclear University MEPhl, 31 Kashirskoe Shosse, Moscow, 115409, Russia

The aim of the investigation was to study the activity of human mirror neurons during observation and perception of short time intervals depending on brain laterality.

Materials and Methods. The study involved young male volunteers aged 18-27 years. During the preliminary examination, the features of brain laterality were investigated determining the dominant hand (by questioning) and the language-dominant hemisphere (dichotic test). EEG was recorded using the 10-20\% system in frontal, central, temporal, parietal, and occipital leads during observation and measurement of short time intervals, observation and reproduction of a five-second rhythm. To study spatial localization of mirror neurons, in some experiments, brain activity was analyzed during observation and time perception using functional magnetic resonance imaging (fMRI). Mu rhythm depression and cortical interactions between the central and other cortical zones at mu frequency were used as EEG markers of mirror neuron activation.

Results. It has been found that observation and execution of actions related to time perception by the subjects are accompanied by EEG mu rhythm depression and, most often, increased levels of cortical connections at mu frequency. The nature of these changes depends on mu frequency, brain laterality, the type and stage of the performed activity. The results of fMRI scanning during observation and time perception show that time perception processes actually involve two systems working together. They are the mirror neuron system including areas of the premotor, motor, sensorimotor and supplementary motor cortex as well as areas of the putamen, the caudate nucleus, the supramarginal gyrus, the temporal lobes of the brain, and the timing system including areas of the callosal gyrus, the left thalamus, the temporal lobes of the brain, the visual cortex, the precuneus and the cerebellum.

The results of the study have significant theoretical importance for understanding the role of mirror neurons in time perception processes.

Key words: time perception in humans; mirror neurons; mu rhythm; cortical interactions; fMRI brain scanning.

\section{Introduction}

Studying the role of mirror neurons in cognitive processes is important for understanding human social behavior. According to the hypothesis popular today [1], mirror neurons can serve as a neuronal basis for the interpretation of actions, imitative learning, and imitation of other people's behavior. Some researchers argue that mirror neuron dysfunction can cause autism [2].

It is believed that the specific EEG-correlate of mirror neuron activation is mu rhythm depression with $8-13 \mathrm{~Hz}$ frequency, which is registered in the central regions of the cortex and is not suppressed during visual and auditory stimulation [3]. However, the studies conducted in this field have yielded mixed results. In particular, when studying electrical activity of the brain at the central and occipital leads in subjects watching hand movement videos and kaleidoscopic patterns on the screen, the authors come to the conclusion that mu rhythm depression can serve as an indicator of human mirror neuron activation, but the effect is weak, unreliable and easily mixed with the occipital alpha rhythm depression [4]. Besides, some researchers [5] distinguish two subbands in the mu rhythm: 8-10 and 10-12 Hz, which are functionally different, in their opinion. A number of studies have found significant individual differences in mu rhythm reactivity during execution of motor tasks [6-9].

Corresponding author: Mikhail V. Svetlik, e-mail: mihasv@ssmu.ru 
All these data point to the need for further investigation of the role of mirror neurons in cognitive processes and, particularly, in time perception processes where their function has been understudied. Research aimed at finding reliable EEG markers of mirror neuron activation, studying the dependence of their activity on individual human characteristics and clarifying their spatial localization is still relevant.

The aim of the investigation was to study the activity of human mirror neurons during observation and perception of short time intervals depending on brain laterality.

\section{Materials and Methods}

To achieve the stated objective, EEG and $\mathrm{fMRI}$ studies of brain activity were performed in young males during their observation and perception of short time intervals.

EEG study involved volunteers, healthy young male students aged 18 to 23 years $(n=31)$. All subjects gave informed consent to participate in the study. The study complies with the Declaration of Helsinki (2013) and was performed following approval by the Bioethics Committee of the Biological Institute of Tomsk State University.

During the preliminary examination by standard methods, the features of brain laterality were investigated with determining the dominant hand (by questioning) and the language-dominant hemisphere (dichotic test). The handedness index and the right-ear coefficient were calculated in scores, using the results of questioning.

Six series of observations were carried out to complete the set tasks. In the first series ("observing the action of measuring a duration"), the subject observed the operator measuring short intervals of time with the middle and index fingers of the dominant hand giving a pause between two presses on the spacebar. The length of time intervals was determined by visual stimuli (a light square appearing in the center of the darkened monitor screen for 200 or $800 \mathrm{~ms}$ ). The stimuli were presented at least 50 times and at random.

In the second series, the subject himself performed the specified action with the fingers of the left hand, and in the third series, he did the same with the fingers of the right hand.

In the fourth series ("observing the action of rhythm reproduction"), the subject observed the operator who first memorized a five-second rhythm, then reproduced the rhythm pressing the spacebar regularly with the index and middle fingers of the dominant hand. The rhythm period was set by visual stimuli (a light square appearing in the center of the darkened screen for $200 \mathrm{~ms}$ ).

In the fifth series, the subject himself performed the specified action with the fingers of the left hand, and in the sixth series, he did the same using the fingers of the right hand.

EEG was recorded before and during execution of actions using the Encephalan-131-03 electroencephalograph (Medikom MTD, Russia) in frontal, central, temporal, parietal, and occipital leads according to the $10-20 \%$ system. To exclude the artifacts associated with eye movement and muscle activity, electrooculography and electromyography of the neck and forehead muscles were performed. When analog signals were input into the computer, the sampling rate was $250 \mathrm{~Hz}$. To study the cortical connections at mu frequency, EEG was pre-filtered. For this purpose, we used a $20^{\text {th }}$ order Butterworth bandpass filter with frequency suppression coefficient of at least $80 \mathrm{~dB}$ for frequencies above $13 \mathrm{~Hz}$ and at least $40 \mathrm{~dB}$ for those below $8 \mathrm{~Hz}$. When processing the data obtained, the maximum values of cross-correlation functions and EEG spectral power were calculated on short segments of EEG recording (1-1.5 s): $3 \mathrm{~s}$ before pressing the key ("background" stage); $1.5 \mathrm{~s}$ before pressing the key ("preparation" stage); immediately after pressing the key ("action execution" stage). The obtained values of correlation coefficients and spectral power level were averaged separately for each stage of the activity, for each series and for all subjects. When calculating cross-correlation functions, we relied on the existing recommendations [10] stating that the maximum time shift should be no more than one tenth of the implementation length which was chosen more than or equal to ten mu rhythm periods (1-1.5 s). The Fourier transform was used to describe the EEG power spectrum. Spectrum calculation was performed with approximation to whole harmonics $(8,9,10,11,12,13 \mathrm{~Hz})$, which made it possible to simplify significantly the subsequent statistical processing and comparative analysis of the results. Statistical data processing was performed using the MatLab v. 6.5 package, non-parametric analysis of variance and the Wilcoxon test for related samples.

The fMRI study involved 10 healthy young volunteers aged 19 to 27 years; the mean age was 23 years. Each subject gave voluntary informed consent to participate in the experiment. Permission to carry out these studies was granted by the Ethics Commission of National Research Center "Kurchatov Institute". During the preliminary examination, the above techniques were used to study the features of brain laterality determining the dominant hand and the language-dominant hemisphere. The fMRI findings were obtained on 3-Tesla Magnetom Verio MRI scanner (Siemens, Germany) at Kurchatov NBICS-complex of the National Research Center "Kurchatov Institute".

The study included several series of experiments. In the first series, which was "observing the action of measuring a time interval", the subject watched a video of the operator's hand whose fingers measured a time interval of $0.8 \mathrm{~s}$ pausing between two presses on the spacebar.

In the second series, "measuring a time interval", the subject being scanned himself measured the specified time interval pressing the button with the left-hand or right-hand fingers, according to the instruction. 
In the third series, "observing the action of rhythm reproduction", the subject was previously shown a video with a white square regularly appearing in the center of the screen (at 5-second intervals). After that, a video was demonstrated showing the operator's hand reproducing the given rhythm by pressing the spacebar with the middle and index fingers.

In the fourth series, "rhythm reproduction", the subject himself reproduced the given rhythm pressing the buttons alternately with the right or left hand, according to the instruction.

All fMRI data were processed using SPM8 software package. Pairwise comparisons were made within each series of the experiment based on Student statistics, individual and mean-group level statistic maps with the significance level of $p<0.001$ were obtained. All statistic maps were applied on the template T1-weighted image and anatomical binding of the active voxels to the ICN Atlas was performed.

\section{Results and Discussion}

The results of studying the electrical activity of the brain during observation and time perception. According to the questioning data, the subjects included right-handed persons - 27, ambidexters - 4, lefthanded -0 . According to the dichotic test results, there were 13 individuals with left-hemispheric language dominance, 3 persons with right-hemispheric language dominance, 15 persons with no identified language lateralization.

The performed studies allowed finding statistically significant changes of EEG spectral power at mu frequencies in different stages of the executed activity. The nature of these changes turned out to depend on the rhythm frequency, brain laterality, the type and stage of the activity. For example, in the series of "observing the action of measuring a duration", there is statistically significant $(p=0.034)$ decrease in EEG spectral power in leads $\mathrm{C} 3$ and $\mathrm{C} 4$ at $8 \mathrm{~Hz}$ frequency at the stage of "action execution" compared to the "background".

In the series of "measuring the duration" with the left hand, statistically significant $(p=0.033)$ decrease in EEG spectral power is observed in leads C4 and $\mathrm{Cz}$ at the same frequency and the same action stage. In the series of "measuring the duration with the right hand", statistically significant $(p=0.015)$ decrease in EEG spectral power is observed at this stage in lead $\mathrm{Cz}$ at $13 \mathrm{~Hz}$ frequency.

In the series of "observing the action of rhythm reproduction", there is statistically significant $(p<0.05)$ decrease in EEG spectral power in lead C3 at frequencies of 9 and $10 \mathrm{~Hz}$ and in lead $\mathrm{C} 4$ at $10 \mathrm{~Hz}$ frequency at the "preparation" stage compared to the "background". At the same time, there are no statistically significant changes in EEG spectral power at mu frequencies at the "action execution" stage compared to the "background". The decrease in the spectral power of the mu rhythm detected at certain frequencies at the stages of "preparation" and "action execution" is most likely to reflect the activation of "motor" mirror neurons [11].

Comparing the series of "rhythm reproduction with the left and right hand" allowed detecting interhemispheric differences in EEG spectral power values at mu frequencies. When reproducing the rhythm with the left hand at the stage of "action execution", EEG spectral power in lead $\mathrm{C} 4$ at $11 \mathrm{~Hz}$ frequency was found to be statistically significantly $(p<0.05)$ lower than this value in the same lead when reproducing the rhythm with the right hand.

The analysis of variance revealed statistically significant influence of the "dominant hand" and "language-dominant hemisphere" factors on the spectral characteristics of mu rhythm based on the stage of the performed activity. In particular, there was found statistically significant $(p=0.041-0.012)$ influence of the "dominant hand" factor on the spectral characteristics of mu rhythm in the series of observing the duration being measured at the stage of "action execution" at 8 and $9 \mathrm{~Hz}$ frequencies in leads $\mathrm{C} 3, \mathrm{C} 4$, and $\mathrm{Cz}$. In the series of measuring the duration with the right hand, the influence of "language-dominant hemisphere" factor was revealed at the "background" stage in lead C3 at 8 and $9 \mathrm{~Hz}$ frequencies $(p=0.004-0.002)$.

The analysis of cortical interactions at the mu rhythm frequency during observation and time perception made it possible to find the dependence of these interactions on the stage and type of the performed activity as well as on brain laterality. In particular, it was found that preparation and execution of actions associated with measuring the duration or reproducing a five-second rhythm were most often accompanied by statistically significant $(p<0.05)$ strengthening of cortical connections between the central and frontal, temporal, parietal and occipital cortex zones as compared to the background. This strengthening of cortical connections is likely to reflect transmission of signals from these zones to the central regions of the cortex where "motor" mirror neurons are located [11].

The dependence of cortical interactions on the stage of the performed activity when observing and reproducing a five-second rhythm is shown in Figure 1.

Moreover, studying the features of cortical interactions that occur when activities are performed with the right or left hand makes it possible to reveal interhemispheric differences in cortical connection levels. In particular, when reproducing the rhythm with the left hand (Figure 1 (b)) there was statistically significant enhancement of right-hemisphere connections, and when reproducing the rhythm with the right hand, left-hemisphere connections were strengthening (Figure 1 (c)).

The results of fMRI measuring the brain activity during observation and time perception. Analysis of fMRI brain scan data obtained during observation and perception of time revealed significant differences 


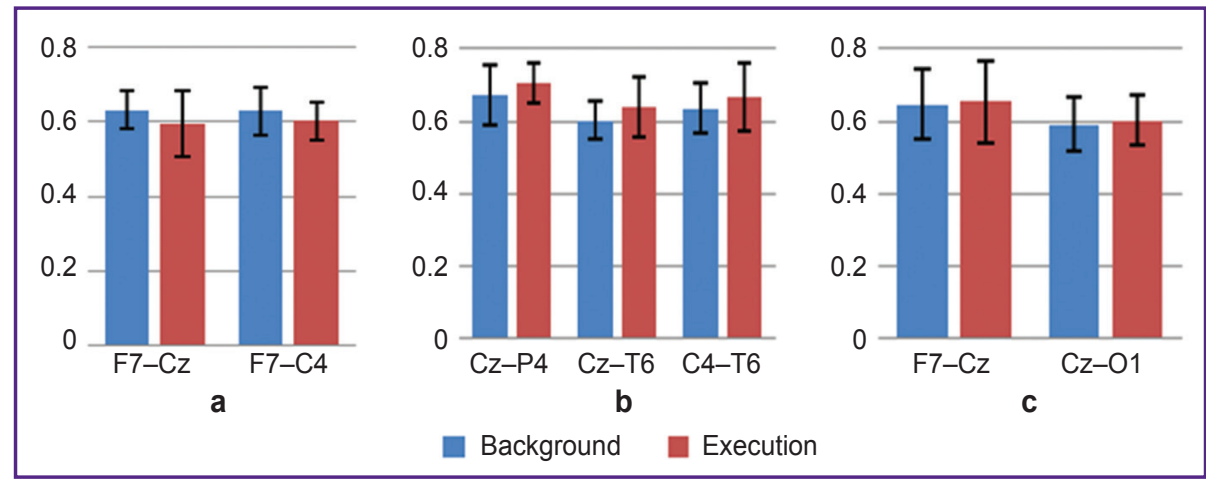

Figure 1. Dependence of cortical interactions at mu frequency on the stage of the activity performed:

(a) in "observation" series; (b) in the series "reproducing the rhythm with the left hand"; (c) in the series "reproducing the rhythm with the right hand". On the ordinate axis, there are correlation coefficient values in relative units. The Figure shows only statistically significant differences $(p<0.05)$

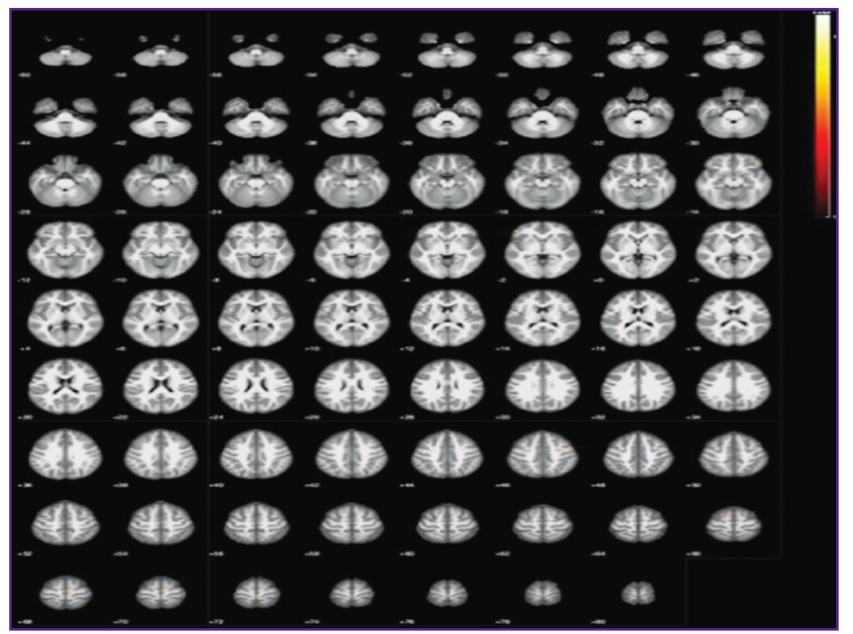

Figure 2. Group-level statistic maps obtained by analyzing series 1 "observing the operator measuring a time interval of $0.8 \mathrm{~s}$ " and applied on the template T1-weighted highresolution image in activation of brain structures during observation and execution of actions associated with time perception.

The results of group analysis of fMRI data for 4 series are presented in Figure 2-5.

In particular, analysis of the results presented in Figure 2 shows that in case when the subject observes the actions of another person measuring a time interval of $0.8 \mathrm{~s}$, the areas of the motor and sensorimotor cortex, the supplementary motor area as well as the putamen and caudate nucleus areas get involved. These zones are known to be mainly responsible for motor activity and can be activated as part of the motor system of mirror neurons when motor movements of another person are being observed [11].

When observing the action of five-second rhythm reproduction (see Figure 3), the areas of the brain related to the supramarginal gyrus are additionally activated. The temporal lobes of the brain and prefrontal cortex areas where "motor" mirror neurons are supposed to be located are also activated [11].

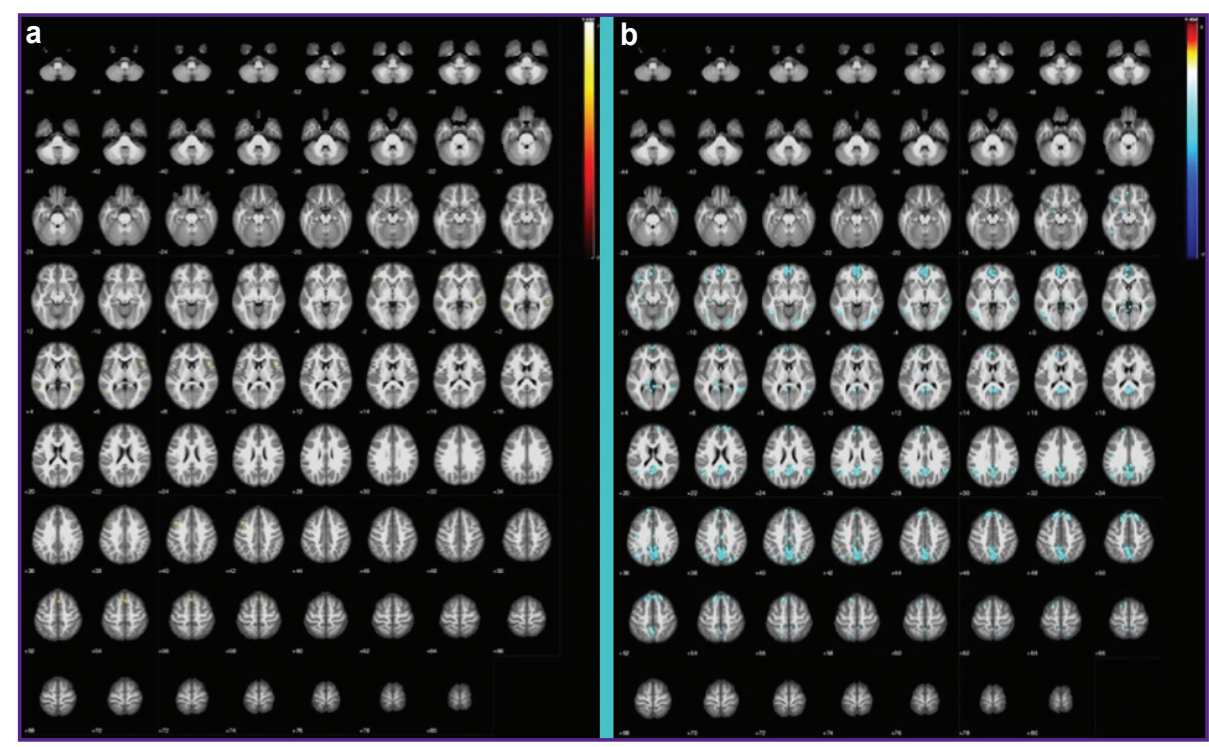

Figure 3. Group-level statistic maps obtained by analyzing series 3 "observing the operator reproducing a five-second rhythm" and applied on the template T1-weighted highresolution image:

(a) comparison of situations "observing the operator reproducing a five-second rhythm" and "rest"; (b) comparison of situations "the operator memorizing a fivesecond rhythm" and "the operator reproducing a five-second rhythm". Comparison of situations "the operator memorizing a five-second rhythm" and "rest" has revealed no statistically significant differences between voxels 


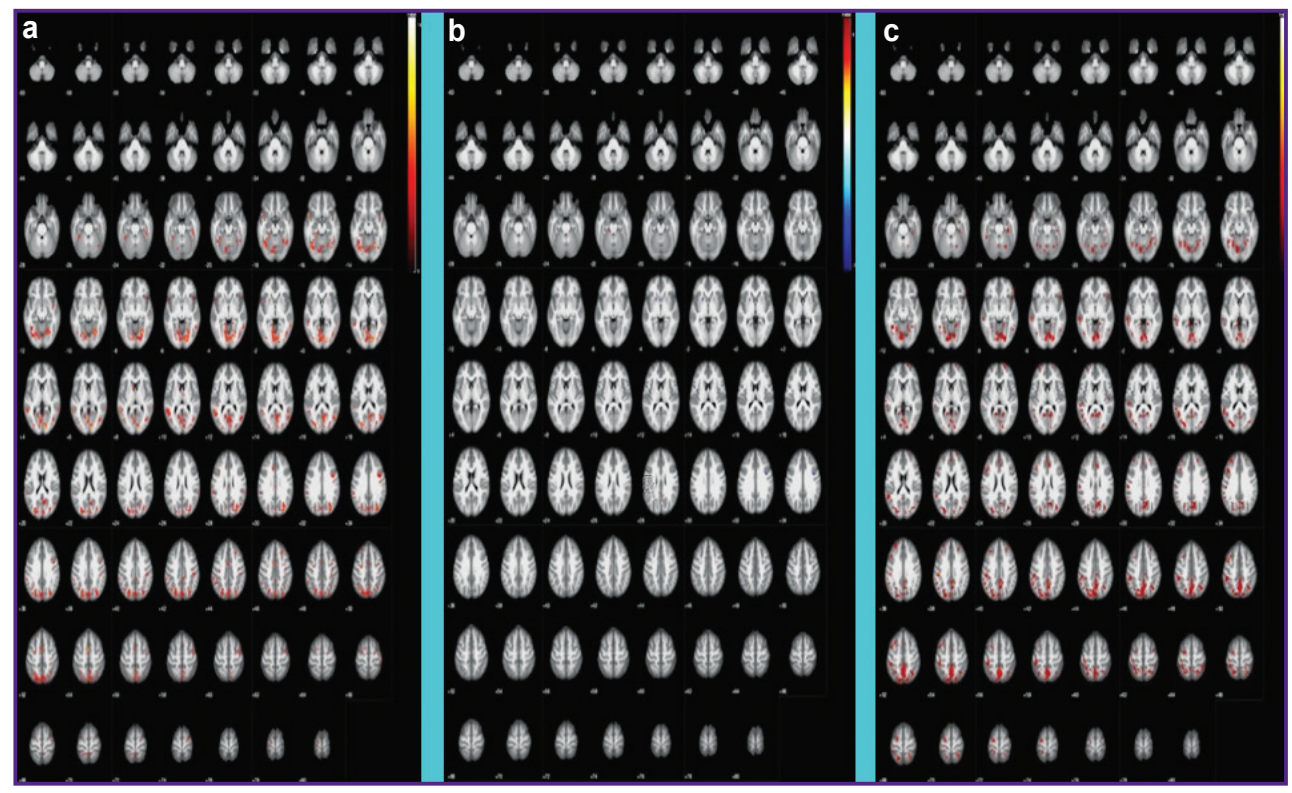

Figure 4. Group-level statistic maps obtained by analyzing series 2 "measuring a time interval of $0.8 \mathrm{~s}$ " and applied on the template T1-weighted high-resolution image:

(a) comparison of situations "measuring a time interval of $0.8 \mathrm{~s}$ with the left hand" and "rest"; (b) comparison of situations "measuring a time interval of $0.8 \mathrm{~s}$ with the right hand" and "measuring a time interval of $0.8 \mathrm{~s}$ with the left hand"; (c) comparison of situations "measuring a time interval of $0.8 \mathrm{~s}$ with the right hand" and "rest"

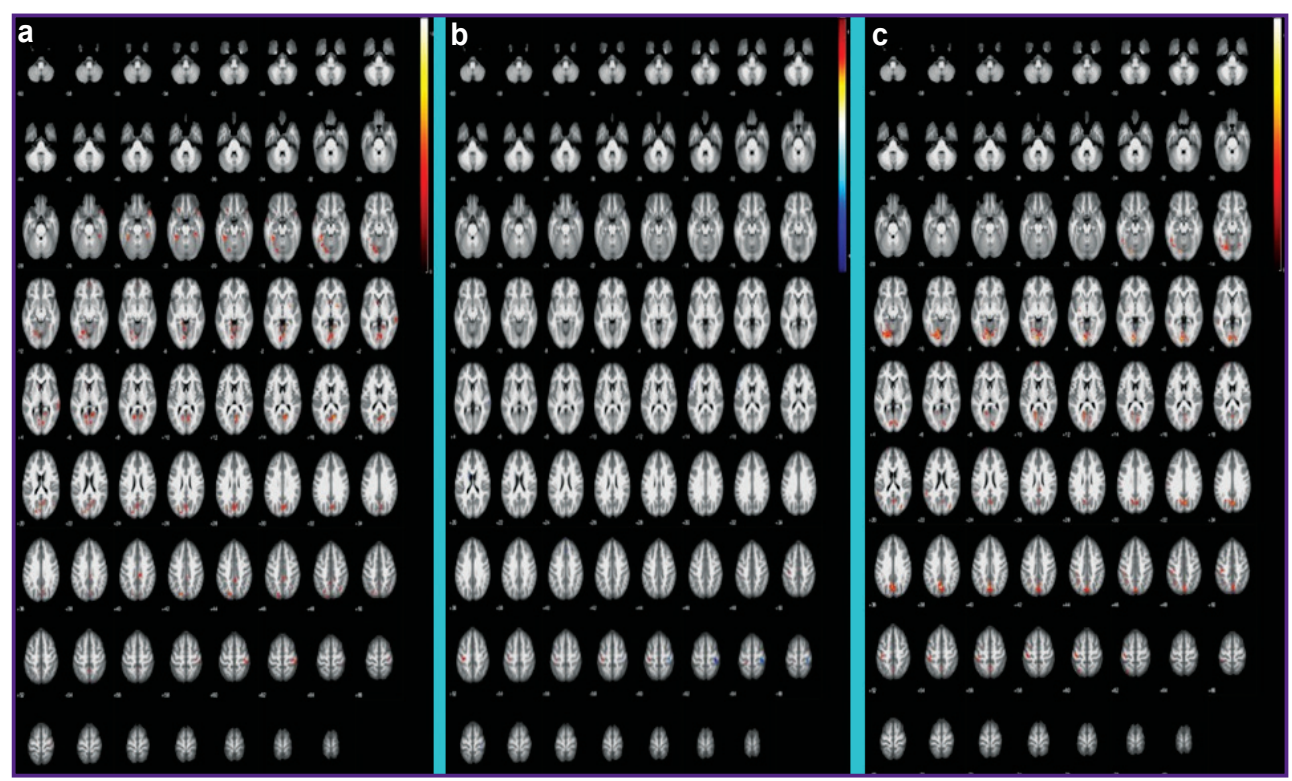

Figure 5. Group-level statistic maps obtained by analyzing series 4 "reproducing a fivesecond rhythm" and applied on the template T1-weighted high-resolution image:

(a) comparison of situations "reproducing a five-second rhythm with the left hand" and "rest"; (b) comparison of situations "reproducing a rhythm with the right hand" and "reproducing a rhythm with the left hand"; (c) comparison of situations "reproducing a rhythm with the right hand" and "rest"

While measuring a time interval of $0.8 \mathrm{~s}$ and reproducing a five-second rhythm (see Figures 4 and 5), the following areas are additionally activated: regions of the callosal gyrus (which might be associated with memory actualization); left thalamus areas; the temporal lobes of the brain; the visual cortex; precuneus regions playing an important role in the processes of consciousness and coordination of movements; regions 
of the cerebellum (left crus 1 and left 6 ) that are involved in the processes of time perception [12].

The obtained results show that the whole complex of brain structures is involved in the processes of time perception. Theoretically, they can be divided into structures related to the mirror neuron system and structures related to the timing system, these systems function together. The first system is likely to include areas of the motor and sensorimotor cortex, the supplementary motor area, zones of the putamen and caudate nucleus as well as areas of the brain belonging to the supramarginal gyrus, which are activated when observing rhythm reproduction and measuring time intervals. It is consistent with some literature data [11] suggesting that the motor mirror system includes neurons of the anterior part of the lower parietal cortex, the lower part of the precentral gyrus as well as the posterior inferior frontal gyrus.

The second system is likely to include zones of the callosal gyrus, areas of the left thalamus, the temporal lobes of the brain, the visual cortex, precuneus regions and the cerebellum (left crus 1 and left 6) which are activated when reproducing a rhythm and measuring time intervals. This is consistent with the literature data indicating that the cerebellum and the basal ganglia [12], the hippocampus [13] as well as the intra-parietal, frontal and auditory cortex $[14,15]$ play an important role in time perception processes.

\section{Conclusion}

Observation and execution of actions associated with time perception are accompanied by mu rhythm depression and, at the same time, increase in the levels of cortical connections at mu frequency. The nature of these changes significantly depends on brain laterality, mu frequency, the type and stage of the executed action.

According to fMRI studies, two systems are actually involved in time perception processes: 1) the mirror neuron system including zones of the motor, the sensorimotor cortex, the supplementary motor area as well as zones of the putamen and caudate nucleus, brain areas belonging to the supramarginal gyrus, the temporal lobes of the brain, prefrontal cortex areas; 2) the timing system including regions of the callosal gyrus, areas of the left thalamus, the temporal lobes of the brain, the visual cortex, areas of the precuneus and the cerebellum.

The results of the investigation are of great theoretical importance for understanding the systemic mechanisms of time perception.

Study funding. This study was supported by the Russian Foundation for Basic Research (grant \#18-01300758) and the internal project of the National Research Center "Kurchatov Institute" (order \#1649 of July 11, 2018).
Conflict of interests. The authors have no conflict of interests to disclose.

\section{References}

1. Skoyles J.R. Gesture, language origins, and right handedness. Psycoloquy 2000; 11(24).

2. Dapretto M., Davies M.S., Pfeifer J.H., Scott A.A., Sigman M., Bookheimer S.Y., lacoboni M. Understanding emotions in others: mirror neuron dysfunction in children with autism spectrum disorders. Nat Neurosci 2005; 9(1): 28-30, https://doi.org/10.1038/nn1611.

3. Alikina M.A., Makhin S.A., Pavlenko V.B. EEG sensorimotor rhythm: amplitude, frequency, topography, age-dependency and functional meaning. Uchenye zapiski Krymskogo federal'nogo universiteta imeni V.I. Vernadskogo. Biologiya. Khimiya 2016; 2(2): 3-24.

4. Hobson H.M., Bishop D.V.M. Mu suppression - a good measure of the human mirror neuron system? Cortex 2016; 82 : 290-310, https://doi.org/10.1016/j.cortex.2016.03.019.

5. Pfurtscheller G., Neuper C., Krausz G. Functional dissociation of lower and upper frequency mu rhythms in relation to voluntary limb movement. Clin Neurophysiol 2000; 111(10): 1873-1879, https://doi.org/10.1016/s13882457(00)00428-4.

6. Yang C.-Y., Decety J., Lee S., Chen C., Cheng Y. Gender differences in the mu rhythm during empathy for pain: an electroencephalographic study. Brain Res 2009; 1251: 176184, https://doi.org/10.1016/j.brainres.2008.11.062.

7. Anwar M.N., Navid M.S., Khan M., Kitajo K. A possible correlation between performance $I Q$, visuomotor adaptation ability and mu suppression. Brain Res 2015; 1603: 84-93, https://doi.org/10.1016/j.brainres.2015.01.045.

8. Höller Y., Bergmann J., Kronbichler M., Crone J.S., Schmid E.V., Thomschewski A., Butz K., Schütze V., Höller P., Trinka E. Real movement vs. motor imagery in healthy subjects. Int J Psychophysiol 2013; 87(1): 35-41, https://doi. org/10.1016/j.ijpsycho.2012.10.015.

9. Makhin S.A., Makaricheva A.A., Lutsuk N.V., Cherniy S.V., Orekhova L.S. Interrelation between individual level of emotional intelligence and EEG sensorimotor rhythm reactivity at the time of synchronized imitation of another person's movement. Uchenye zapiski Tavricheskogo natsional'nogo universiteta imeni V.I. Vernadskogo. Seriya "Biologiya, khimiya" 2013; 26(4): 121-131.

10. Bendat J.S., Piersol A.G. Random data: analysis and measurement procedures. Wiley; 2010.

11. Rizzolatti G., Sinigaglia C. Mirrors in the brain: how our minds share actions and emotions. New York: Oxford University Press; 2008.

12. Jueptner M., Rijntjes M., Weiller C., Faiss J.H., Timmann D., Mueller S.P., Diener H.C. Localization of a cerebellar timing process using PET. Neurology 1995; 45(8): 1540-1545, https://doi.org/10.1212/wnl.45.8.1540.

13. Mering T.A. On different forms of time reflection by the brain. Voprosy filosofii 1975; 7: 119-127.

14. Leon M.I., Shadlen M.N. Representation of time by neurons in the posterior parietal cortex of the macaque. Neuron 2003; 38(2): 317-327, https://doi.org/10.1016/s08966273(03)00185-5.

15. Sysoeva O.V., Vartanov A.V. Reflection of stimulus duration in characteristics of evoked potential (part 1). Rsikhologicheskii zhurnal 2004; 25(1): 101-110. 